\title{
Multi-scale standardized spectral mixture models
}

\author{
Christopher Small ${ }^{\mathrm{a}, *}$, Cristina Milesi ${ }^{\mathrm{b}}$ \\ a Lamont Doherty Earth Observatory, Columbia University, Palisades, NY 10964, USA \\ b California State University Monterey Bay/NASA Ames Research Center, Moffett Field, CA 94035, USA
}

\section{A R T I C L E I N F O}

\section{Article history:}

Received 19 January 2013

Received in revised form 19 May 2013

Accepted 25 May 2013

Available online $\mathrm{xxxx}$

\section{Keywords:}

Spectral mixture model

Landsat

WorldView-2

Scale

Vegetation index

\begin{abstract}
A B S T R A C T
Linear spectral mixture models can be standardized by using endmembers that span the global mixing space. By combining the benefits of location-specific mixture models with standardized spectral indices, standardized mixture models offer consistency, simplicity, inclusivity and applicability. We construct a globally representative mixing space using a spectrally diverse collection of 100 Landsat ETM+ (Thematic Mapper \& Enhanced Thematic Mapper+) subscenes. Global composites of 100,000,000 Landsat spectra, constructed from both exoatmospheric reflectance and atmospherically corrected surface reflectance, represent the spectral diversity of a wide range of terrestrial environments. Principal Component (PC) Analysis of the global composite shows that $99 \%$ of the spectral variance can be represented in a 3-dimensional mixing space of the low order PCs. Within this 3D space $98 \%$ of spectra are contained within a tetrahedral hull bounded by a continuous plane of substrates, and well-defined apexes corresponding to vegetation and dark endmembers. Suites of individual substrate, vegetation and dark endmember spectra are used to derive mean endmembers and to quantify the effects of endmember variability on fractions estimated from a standardized Substrate, Vegetation, and Dark (SVD) linear mixture model. Maximum endmember variability introduces less than 0.05 difference in S, V, and D fractions for most SVD models constructed from individual pixel endmember spectra giving less than 0.05 model misfit for more than $97 \%$ of pixels in the global composite. The mean SVD endmembers define a standard global mixture model for Landsat spectra. These SVD endmembers can be used to model mixed reflectance spectra from other sensors with similar spectral responses to Landsat ETM +. Comparisons of endmember fractions estimated from coincident acquisitions of Landsat TM and ETM + and WorldView-2 imagery show strong linear scaling for vegetation and dark fractions. Substrate fractions do not scale as linearly for the urban validation sites because the Landsat substrate endmember does not accurately represent the impervious surfaces imaged by WorldView-2. Comparisons of Landsat and WorldView-2 unmixed with the same Visible-Near Infrared (VNIR) endmembers derived from the global Landsat endmembers are also strongly correlated but with reduced bias. This linear scaling suggests that the Landsat global endmembers may provide a basis for standardized mixture models for WorldView-2 and other broadband sensors with spectral response similar to Landsat TM and $\mathrm{ETM}+$. Comparisons of vegetation fractions with vegetation indices for the global composite show strong linear correspondence for Tasseled Cap Greenness and Enhanced Vegetation Index, with some degree of saturation at high fractions for the Soil Adjusted Vegetation Index and a wide range of responses for the Normalized Difference Vegetation Index.
\end{abstract}

(c) 2013 Published by Elsevier Inc.

\section{Introduction}

The linear spectral mixture model represents radiance measurements as linear mixtures of endmember radiances reflected from different materials in the sensor's Instantaneous Field of View (IFOV). In cases of homogeneous target spectra, these endmembers are often considered "spectrally pure" but a more general criteria of "spectrally distinct" allows the mixture model to be used in situations where characteristic combinations of materials function as endmembers bounding continua of other spectral mixtures. Inverting the linear mixture model yields per pixel endmember fractions which can be interpreted as quantitative

\footnotetext{
* Corresponding author. Tel.: + 13475354274.

E-mail address: csmall@columbia.edu (C. Small).
}

estimates of the areal abundance of specific land cover types (endmembers) contributing to the mixed pixel (Adams et al., 1986, 1993; Gillespie et al., 1990; Smith et al., 1990). By representing each pixel as a combination of endmembers, the resulting fraction images provide continuous field representations of the spectrally heterogeneous gradations in land cover that characterize much of the Earth surface. For many applications, such as physical models of land surface dynamics, a continuous field corresponding to a physical quantity (e.g., vegetation abundance) can more accurately represent land surface properties than a homogeneous thematic land cover class with discrete boundaries. In applications where distinct thematic classes are required, endmember fractions can be grouped into intervals to provide more physically consistent definitions of thematic classes than may be obtained from statistical classification methods. 
Linear mixture models are usually application-specific in the sense that the model is designed with spectral endmembers specific to the location or problem at hand. The number and choice of endmembers are the defining characteristics of the model. However, it is also possible to use the linear mixture model as a more general representation of land cover by using generic endmembers representative of common land cover types. We refer to a general linear mixture model, based on generic spectral endmembers, as a standardized spectral mixture model. A standardized spectral mixture model can offer many of the benefits of a spectral index (e.g., vegetation index) while providing a simple, physically-based representation of the abundance of different materials within the IFOV. The implicit assumption is that non-linear mixing (e.g., from multiple scattering) is negligible. In order to be generally applicable, the standardized spectral mixture model must represent the diversity of materials likely to be imaged at different locations and times. This means that the number and choice of generic endmembers that define the model must encompass the range of reflectances that can be distinguished by the sensor. The standardized spectral mixture model does not imply that the endmembers used are the only spectrally distinct (i.e., distinguishable) endmembers that exist. It merely represents the mixed reflectance measurement as the combination of generic endmember fractions that most closely matches the measurement. The standardized mixture model is not intended to replace location or problem-specific mixture models but rather to supplement them and to allow fractions to be compared consistently across locations and time.

A standardized spectral mixture model can be thought of as an alternative coordinate system within which a continuum of spectral mixtures can be represented in terms of a small number of canonical endmembers representing the most spectrally distinct land cover components that the sensor can resolve (Small, 2004b). These canonical endmembers are analogous to the "universal endmembers" discussed by Adams and Gillespie (2006) for specific types of scenes - but are further generalized to represent the diversity of spectral mixtures that can be resolved by a given sensor over the full range of landscapes found on Earth. An important benefit of this alternative coordinate system is potentially a lower dimensionality than that defined by the (possibly redundant) bands of the sensor. Another benefit is the ability to represent landscapes as continuous fields of fundamental land cover components. The standardized spectral mixture model has its conceptual origin in the Kauth-Thomas model of spectral evolution of agricultural landscapes (Kauth \& Thomas, 1976) but includes all landscapes for which the important components can be resolved by the sensor being used. Despite the conceptual similarity in their origins, there are important distinctions between the standardized linear spectral mixture model and the Kauth-Thomas Tasseled Cap Transformation (TCT) as explained in the Discussion below.

This depiction of a standardized linear spectral mixture model, and its distinction from the underlying physical concept of spectral mixing in the radiance field raises two complementary points. 1) The image endmembers that a particular sensor can distinguish do not necessarily encompass all spectrally distinct materials that might be considered endmembers for a different sensor capable of distinguishing more or different spectra. In this sense, the mixture model is sensor-specific. 2) Different sensors with similar spectral responses can represent the same target reflectance similarly. This suggests that generic endmembers derived from one sensor may provide a basis (literally and mathematically) for linear mixture models of spectra measured by other sensors with similar spectral responses. In this sense, the mixture model and its canonical endmembers may be portable from one sensor to another.

The objectives of this study are 1) to characterize the topology and spectral dimensionality of the Landsat ETM+ spectral mixing space, 2 ) to identify spectral endmembers that span the space, 3 ) to quantify image endmember variability and its effect on the distribution of misfit to the standardized mixture model, 4 ) to quantify the linearity of spatial scaling of fractions derived from the generic mixture model and 5) to compare vegetation fractions from the standardized mixture model with other standardized vegetation metrics over a wide range of different environments. As the basis for the analysis we use a global composite of 100 spectrally diverse subscenes collected by Landsat 5 and Landsat 7. We use the abbreviation ETM+ to refer to intercalibrated imagery collected by either the TM or ETM + sensor. The images are calibrated to both exoatmospheric reflectance (Chander et al., 2009) and surface reflectance (Masek et al., 2006) to yield endmembers for each type of calibration. To accomplish these objectives we first construct a global composite from the 100 subscenes and select suites of Substrate, Vegetation, and Dark (SVD) endmembers spanning its 3D mixing space. We use these endmember suites to quantify the effect of endmember variability on the SVD endmember fractions estimated for the global Landsat composite. We investigate the linearity of spatial scaling by comparing endmember fractions derived from Landsat with fractions derived from near simultaneous acquisitions of WorldView-2. Finally, we compare vegetation fractions estimated with the generic endmembers to Tasseled Cap greenness and three vegetation indices for the global composite to illustrate the relationships between them in a wide variety of environments.

This study uses the analysis of Small (2004b) as a starting point and extends the analysis in five ways. 1) The original set of 30 subscenes is expanded to a larger, more geographically diverse collection of 100 subscenes of both Landsat 5 TM and Landsat 7 ETM +. 2) Parallel analyses are conducted using both exoatmospheric (top of atmosphere) and atmospherically corrected surface reflectance. 3) Effects of endmember variability on fraction distributions are quantified. 4) Linearity of spatial scaling of endmember fractions from $2 \mathrm{~m}$ to $30 \mathrm{~m}$ is demonstrated. 5) Vegetation fraction estimates from the standardized model are compared to other standardized vegetation metrics for the diverse range of environments in the global Landsat composite.

\section{Data}

A spectrally diverse collection of 100 subscenes from 67 unique Level 1 terrain corrected (L1T) Landsat ETM + scenes was selected on the basis of diversity of land cover and diversity of biomes (Fig. 1). The global collection spans all terrestrial biomes as determined by mean annual temperature and precipitation (Houghton et al., 1996) in approximate proportion to land area (Small, 2004a). The DNs are calibrated to exoatmospheric reflectance using the calibration approach and coefficients given by Chander et al. (2009). We also convert the data to surface reflectance correcting for atmospheric effects by means of the $6 \mathrm{~S}$ code implementation in the Landsat Ecosystem Disturbance Adaptive Processing System (LEDAPS) atmospheric correction method (Masek et al., 2006), which is currently used by the United States Geological Survey to distribute surface reflectance Landsat data. Ice sheets and open marine environments are not well represented in the collection because the atmospheric correction is known to be problematic over these surfaces. In each scene we strive to use cloud-free imagery to the extent possible. The atmospheric correction reduces the perturbations caused by the Rayleigh scattering and the absorption of the mixing atmospheric molecules and aerosols (Vermote et al., 1997). In the analyzed dataset, the LEDAPS correction (ledapsSrc.20111121) acts primarily on reducing the effects of Rayleigh scattering at low reflectances of the visible bands and increasing the reflectance in the SWIR, which is otherwise reduced by aerosols and other gas molecules absorption (Ju et al., 2012). In our study the atmospheric correction has the effect of eliminating some of the random variations in the fractions that would otherwise appear from unmixing exoatmospheric reflectances. A comparison of the atmospherically corrected reflectances to the exoatmospheric reflectances shows the two variables to be strongly collinear with absolute differences less than 0.07 for more than $98 \%$ of pixels in all bands, indicating a reasonable performance of the LEDAPS code. For each of the 67 Landsat scenes used, one to four $30 \times 30 \mathrm{~km}$ subscenes were chosen on the basis 


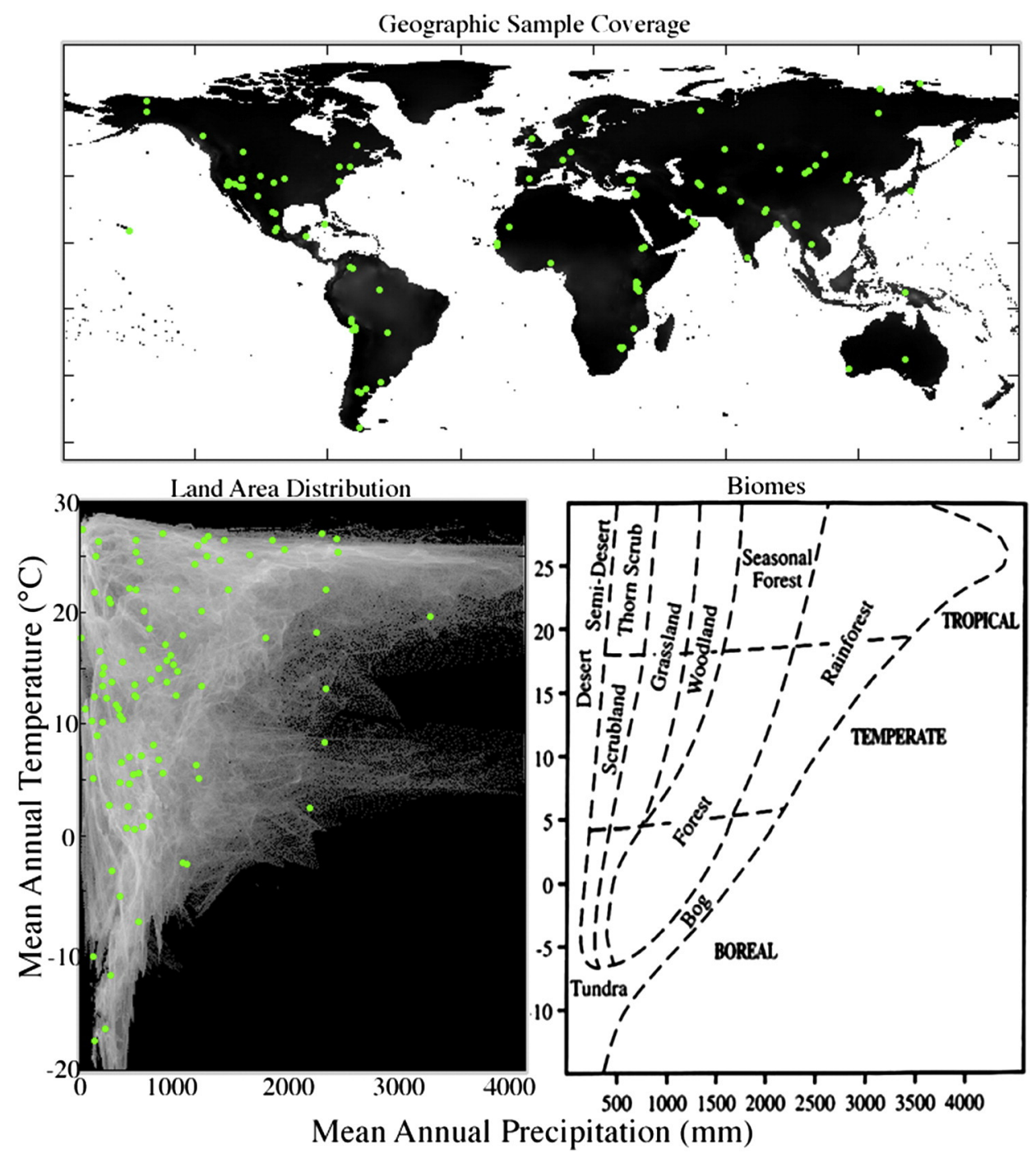

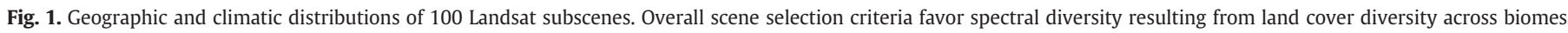

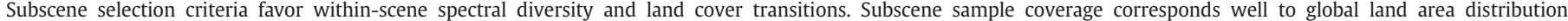

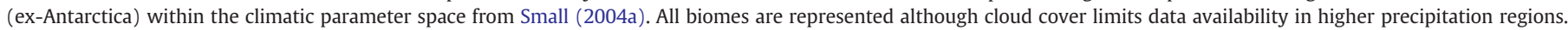
Biome classification modified from Houghton et al. (1996). Ice sheets are omitted because of atmospheric correction limitations.

of spectral diversity. The 100 subscene collection includes all of 30 subscenes used by Small (2004b). For each reflectance product all 100 subscenes were combined into a separate composite image referred to as the global composite.

The global composite illustrates both the spectral diversity and the consistency of land cover as imaged by Landsat (Fig. 2). When all 100 subscenes are displayed with the same linear stretch applied to a Visible/Near Infrared/Short Wave Infrared composite (bands 2,4,7) the dominance of substrates (brown), vegetation (green) and water (black) is apparent. Shallow marine substrates, evaporates (playas, dry lakes and other surfaces containing partially hydrous precipitate deposits), ice and snow all have comparatively high visible reflectance (relative to IR) and therefore appear in shades of blue to cyan. When each subscene has a $2 \%$ linear stretch applied separately, the spectral diversity of each is more apparent. The dates and locations of each subscene, along with full-resolution false color composites are available online at: http:// www.LDEO.columbia.edu/ small/GlobalLandsat/.

We test linearity of scaling by comparing $30 \mathrm{~m}$ Landsat fraction estimates from global endmembers with high resolution $(2 \mathrm{~m})$ fraction estimates for two pairs of coincident Landsat and WorldView-2 (WV2) acquisitions. New York City (NYC) is chosen for the test site because of its spectral diversity and because we have two clear sky WV2 acquisitions coinciding with same day Landsat acquisitions. WV2 imaged NYC 23 min after Landsat 7 on 4/23/2010 and 32 min after Landsat 5 on $5 / 1 / 2010$. On the later acquisition, the WV2 view geometry was very similar to that of Landsat 5 but on the earlier acquisition the elevation angles differ by $17^{\circ}$.

\section{Analysis}

We use a Principal Component (PC) analysis to quantify the spectral dimensionality of the global composite and to render the mixing space from which the endmembers are selected. We infer spectral dimensionality from the variance partition given by the eigenvalues of the covariance matrix of the spectral bands of the global composite. By dimensionality we refer to variance distribution - not necessarily information content. After rendering the mixing space from the three low order PCs we identify a suite of candidate endmember spectra from the apexes of the pixel cloud corresponding to substrates (S), vegetation $(\mathrm{V})$, and dark surfaces (D). We refer to the $S, V$, and $D$ endmembers as primary endmembers because they bound the vast majority of pixels in the mixing space and represent the fundamental 
Common Linear Stretch [0.0, 0.5]

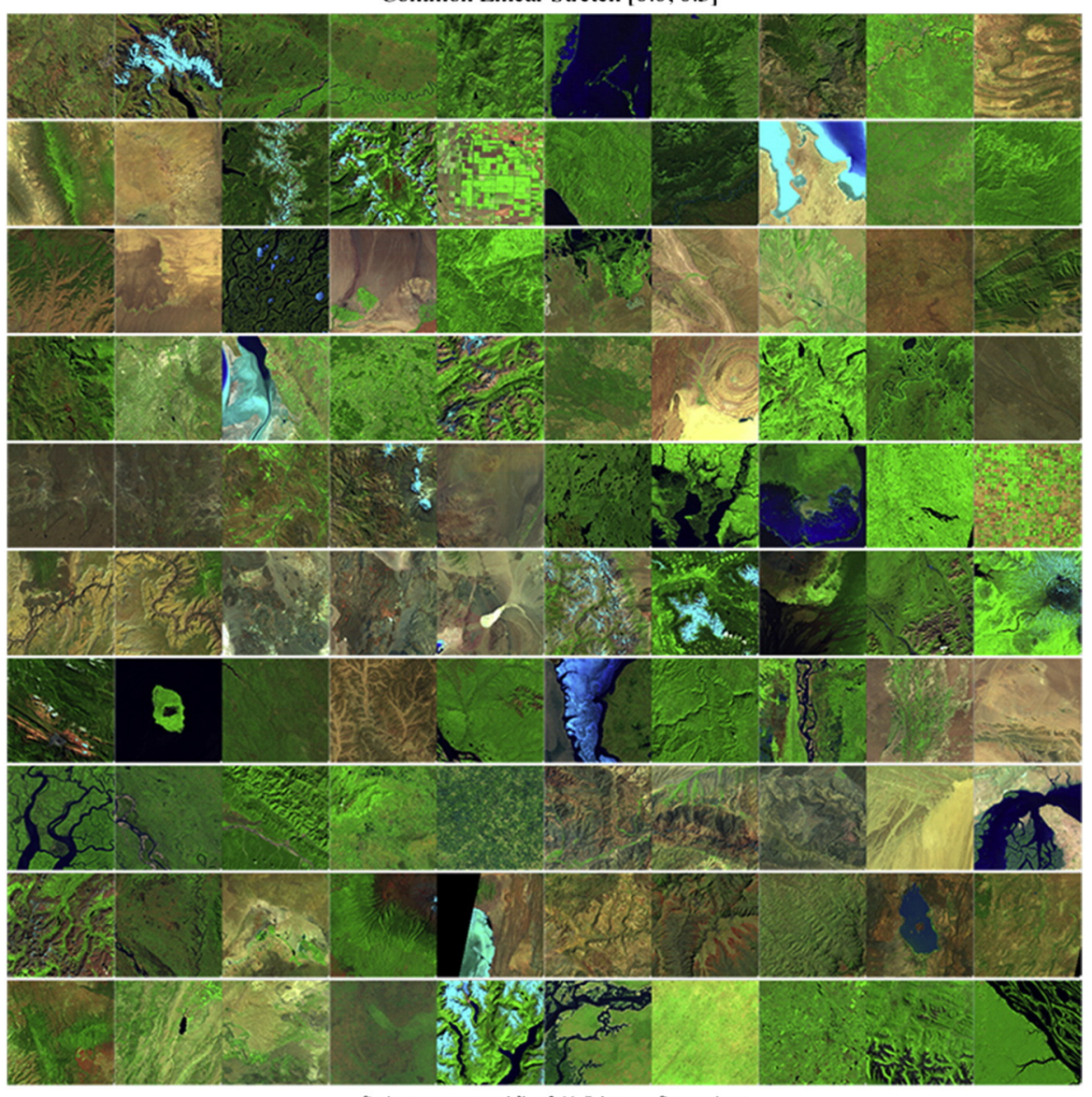

Subscene-specific 2\% Linear Stretches

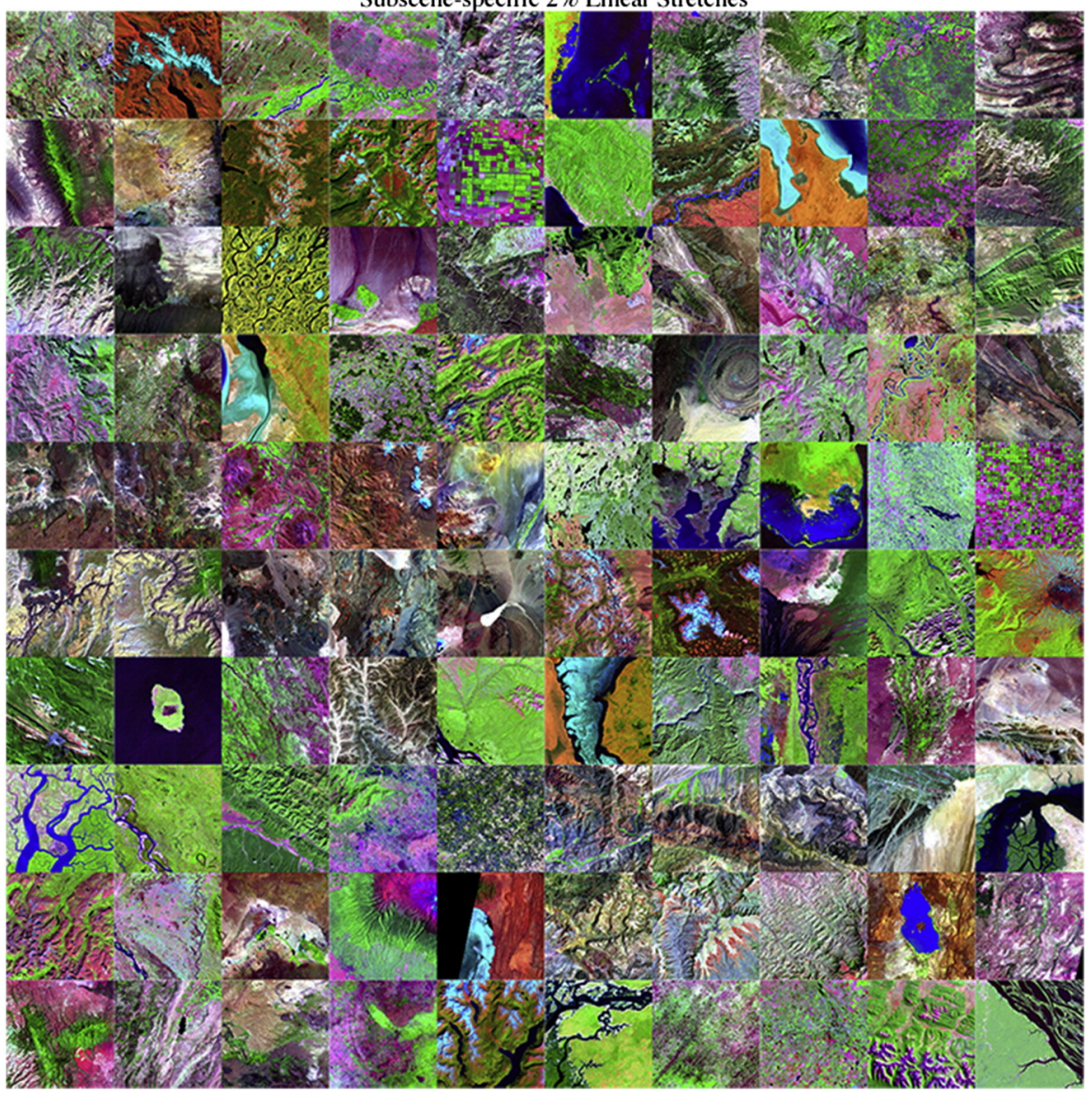


Table 1

Global endmembers calculated for the exoatmospheric reflectances (subscript E) and surface reflectances (subscript S).

\begin{tabular}{lllllll}
\hline$\lambda \mu \mathrm{m}$ & Substrate $_{\mathrm{E}}$ & Vegetation $_{\mathrm{E}}$ & Dark $_{\mathrm{E}}$ & Substrate $_{\mathrm{S}}$ & Vegetation $_{\mathrm{S}}$ & Dark $_{\mathrm{S}}$ \\
\hline 0.479 & 0.479 & 0.211 & 0.093 & 0.178 & 0.030 & 0.019 \\
0.561 & 0.317 & 0.087 & 0.044 & 0.337 & 0.060 & 0.010 \\
0.661 & 0.427 & 0.050 & 0.026 & 0.458 & 0.031 & 0.005 \\
0.835 & 0.525 & 0.611 & 0.017 & 0.559 & 0.669 & 0.007 \\
1.650 & 0.623 & 0.220 & 0.005 & 0.683 & 0.240 & 0.003 \\
2.208 & 0.570 & 0.080 & 0.003 & 0.645 & 0.096 & 0.002 \\
\hline
\end{tabular}

physical components of most land surfaces not covered by water, ice or snow. The names Substrate, Vegetation, and Dark are chosen for brevity. In reality, Substrate includes rock, sediment, soil and non-photosynthetic vegetation (NPV). Vegetation refers to photosynthetic foliage characterized by chlorophyll absorptions in the visible and high reflectance in the NIR. The Dark endmember contains a fundamental ambiguity resulting from low surface reflectance and the dominance of atmospheric scattering. Dark targets may be either absorptive (e.g., black lava), transmissive (e.g., deep clear water) or non-illuminated (shadow). The reflectances used here are distinct from reflectivity in their dependence on solar illumination and surface roughness. We also identify secondary endmembers representing specific deviations from the plane spanned by the $S, V$, and $D$ endmembers to illustrate the spectral character of these deviations.

After selecting endmember suites we invert (unmix) a simple threeendmember linear mixture model to estimate Substrate, Vegetation, and Dark fractions. The model is inverted using averaged endmembers as well as individual pixel endmember spectra to quantify the effects of endmember variability as described below. All SVD models are inverted using the least squares solution (Settle \& Drake, 1993) with a unit sum constraint of equal weight to the band-specific fraction sum equations. For each inversion the endmembers and the estimated endmember fractions are used to forward model the mixed reflectances for comparison to the observed reflectances. The Root Mean Square (RMS) difference between the observed and modeled mixtures is then used as a metric to quantify model misfit and the effects of endmember variability.

We quantify the potential effects of endmember variability on the endmember fraction estimates by unmixing the global composite with different combinations of individual pixel endmember spectra. We distinguish here between image endmember variability and spectral variability of endmember materials. We quantify the effects of image endmember variability among the individual pixel spectra that reside near apexes of the pixel cloud in the mixing space. This is related to, but distinct from, natural spectral variability of materials and land cover types that may serve as endmembers. Both types of variability, and methods to minimize their effect on model results, are discussed in detail by (Somers et al., 2011). Fraction sensitivity to endmember variability is quantified here by comparing RMS misfit distributions and fraction difference distributions for all combinations of individual pixel endmember spectra. We acknowledge that RMS is a simple metric and may underrepresent some spectral distinctions but assume that it will capture the most important deviations between models. In practice, the residual (i.e., RMS misfit) images and individual band residuals can provide more detailed information on the disparities between the modeled and observed spectra which can be used to improve the model. The maximum number of distinct individual pixel spectra in the vicinity of the S, V or D apex was for the substrate endmember, which had 9. For the vegetation and dark endmembers we included additional endmember spectra with multiple pixel occurrences so as to

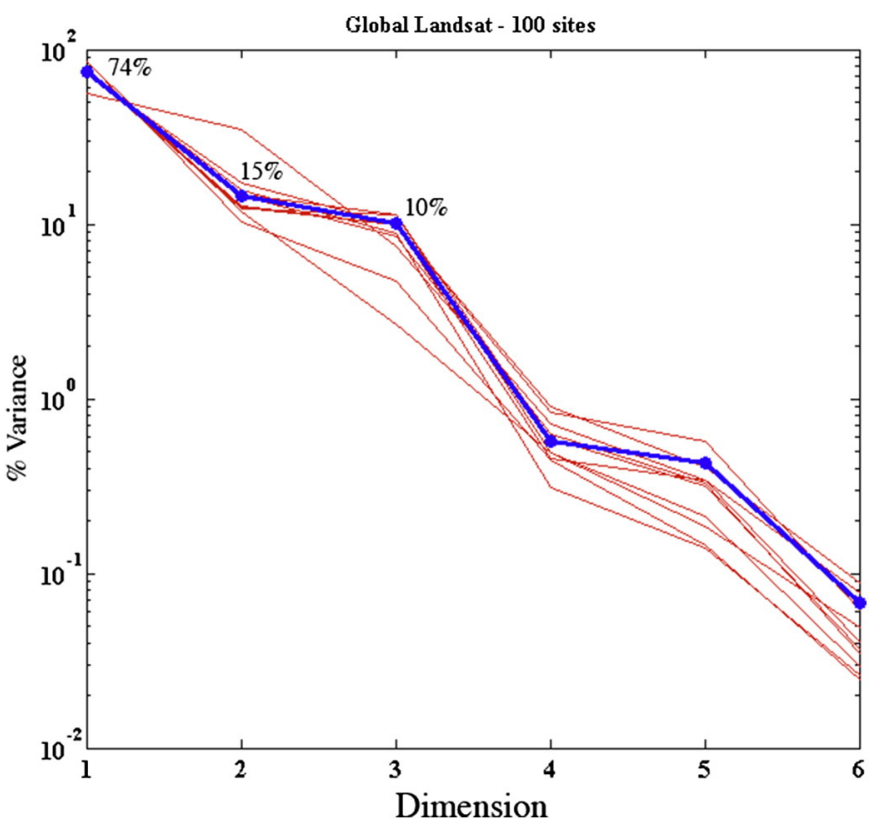

Fig. 3. Spectral dimensionality of the 100 Landsat ETM+ subscenes. Eigenvalues of the global composite covariance matrix show similar variance distributions for 10 subsets of 10 subscenes each (red) and for all 100 subscenes together (blue). Three low order dimensions account for at least $98 \%$ of variance in all cases. Although the Landsat feature space is 6 dimensional, the eigenstructure of the mixing space is effectively 3 dimensional. (For interpretation of the references to color in this figure legend, the reader is referred to the web version of this article.)

have 9 spectra per endmember suite for comparison. Within the set of convex hulls defined by these triplets of individual pixel spectra, the pixel density of the mixing space increases and the similarity among adjacent pixel spectra increases (Fig. 3).

The objective of comparing the individual pixel endmember models is to quantify how endmember variability may propagate into fraction distributions. Rather than unmix 729 (9 spectra $\wedge 3$ endmembers $=9^{3}$ ) models, we select the two most different pixel spectra for each endmember and compare the $8\left(2^{3}\right)$ SVD models that encompass the maximum variability of each endmember suite. For each $\mathrm{S}, \mathrm{V}$, and D suite of 9 individual pixel endmember spectra we identify the two most disparate spectra as those which have the greatest RMS difference out of all $36(8+7+6+5+4+3+2+1)$ pairs of candidate spectra for that endmember. The 2 most disparate $\mathrm{S}, \mathrm{V}$, and $\mathrm{D}$ spectra ( 6 total) provide the basis for 8 linear mixture models $(2 \times 2 \times 2$ permutations) of the global composite. The 8 disparate models allow for 28 model-to-model comparisons. The differences among the 28 $(7+6+5+4+3+2+1)$ pairwise permutations of each $\mathrm{S}, \mathrm{V}$, and $\mathrm{D}$ endmember fraction provide an indication of how endmember variability maps into differences in the fractions resulting from different combinations of the individual endmember spectra. We consider both RMS misfit distribution of the 8 SVD models and fraction difference distributions for each of 28 pairwise differences of $\mathrm{S}, \mathrm{V}$, and $\mathrm{D}$ endmember fractions for the global composite. Because the effects of endmember variability are found to be generally small, and because the use of any individual pixel spectrum would be difficult to justify, we use the mean of each suite of 9 candidate spectra as the standard endmembers for the SVD model. The assumption that all 9 individual pixel spectra are equally valid is supported by the low overall variability in the spectra of each endmember. The fraction variability provides some indication of how

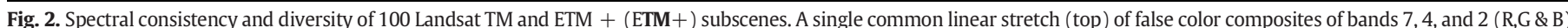

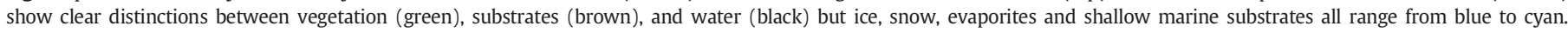

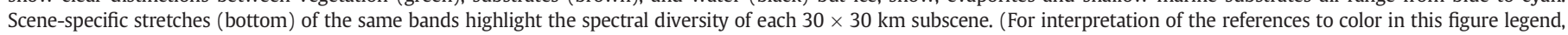
the reader is referred to the web version of this article.) 
endmember variability corresponds to uncertainty in fraction estimates for a diverse distribution of spectra. It also gives an indication of what kind of error distribution might be expected to result from using individual pixel endmembers rather than averages.

To quantify the linearity of spatial scaling of fraction estimates we compare $30 \times 30 \mathrm{~m}$ fractions derived from Landsat with aggregated $2 \times 2 \mathrm{~m}$ estimates derived from WV2 imagery. Because the Blue, Green, Red and NIR1 bands of WV2 have similar spectral responses to the corresponding Landsat bands, the VNIR reflectances of the Landsat endmembers can also be used as standard endmembers for unmixing WV2 imagery. We use the vicarious validation approach described by (Small \& Lu, 2006) to compare S, V, and D fractions from four near-simultaneous acquisitions of Landsat and WV-2 for four spectrally diverse areas of New York City. While vicarious validation is not equivalent to in situ validation, it does illustrate the linearity of scaling at a resolution sufficient to oversample individual land cover components. We verify linearity of scaling from $2 \mathrm{~m}$ to $30 \mathrm{~m}$ using scatterplots and difference distributions of coregistered Landsat and WV-2 fractions. First, we compare 8-band WV2 exoatmospheric SVD fractions estimated with local image endmembers to 6-band Landsat surface reflectance SVD fractions estimated with the global Landsat endmembers. Then, synthetic global SVD endmembers for WV2 are derived from four Landsat VNIR bands of the Landsat endmembers. Using these synthetic global endmembers, we invert the generic SVD model for WV-2 exoatmospheric reflectance and compare the resulting fractions with those derived from the corresponding Landsat 5 and Landsat 7 exoatmospheric reflectances using the same approach used for the previous comparison. As a final illustration of the standardized spectral mixture model we compare vegetation fractions with three vegetation indices and Tasseled Cap Greenness for the global composite.

\section{Results}

\subsection{Dimensionality and topology of the global mixing space}

The spectral dimensionality of the global composite can be inferred from the variance partition given by the eigenvalues of the covariability (correlation or covariance) matrix. The implicit assumption is that variance corresponds to unique information contained in the spectral bands. A standardized PC rotation using the correlation matrix produced nearly identical results to those obtained using the covariance matrix; subsequent analysis uses the covariance-based statistics. Fig. 3 shows similar variance partition for ten subsets of ten subscenes each and for the entire global composite. In all cases, at least $98 \%$ of variance is associated with the three low order dimensions of the mixing space. Although the Landsat mixing space has 6 dimensions, in terms of variance it is effectively three dimensional (Small, 2004b). For this reason, we infer the topology of the mixing space from the three low order PCs. We acknowledge that the remaining high order dimensions may contain very useful information despite the low variance. Because the focus of this study is on the utility of the SVD model, we will consider the unmodeled residuals of the SVD model in a separate study.

The topology of the 3D mixing space is dominated by ternary mixtures of substrates, vegetation, and dark surfaces (Fig. 4). The mixing spaces of the exoatmospheric and surface reflectances are indistinguishable from one another. Visible-bright spectra like evaporates, ice, snow, reefs and shallow marine substrates form distinct binary mixing

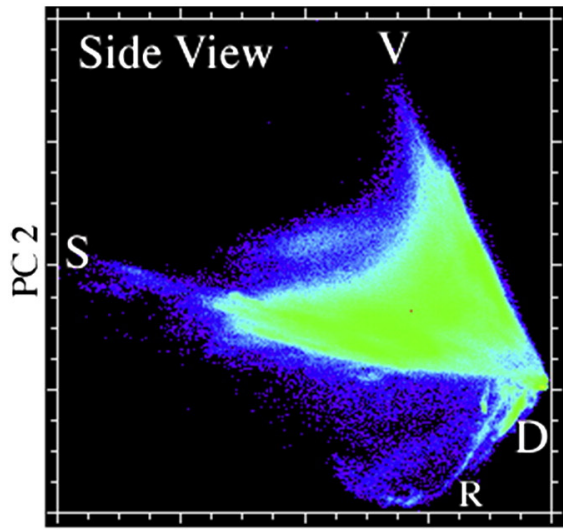

PC 1

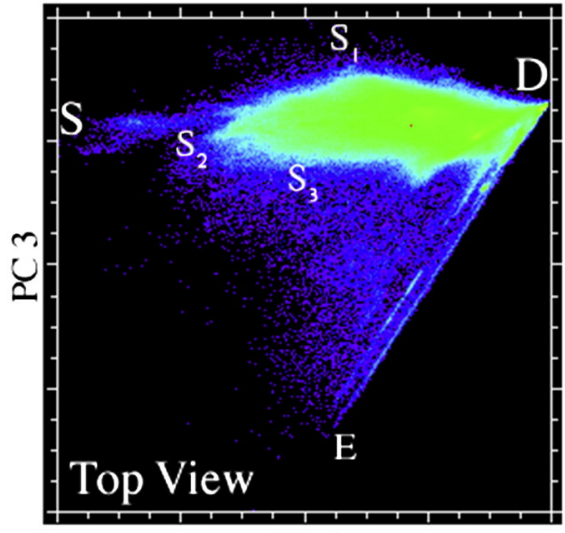

PC 1

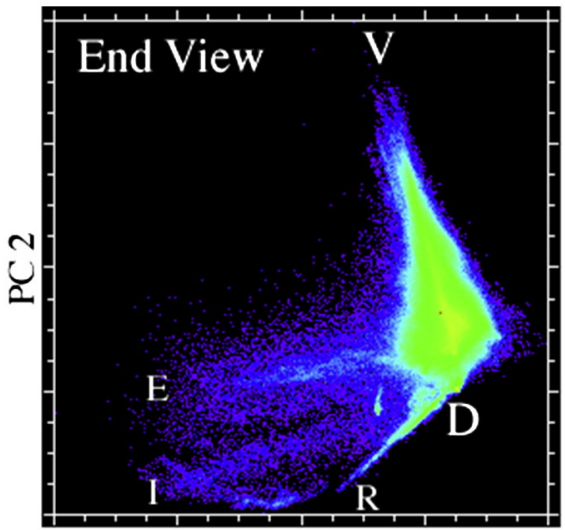

PC 3

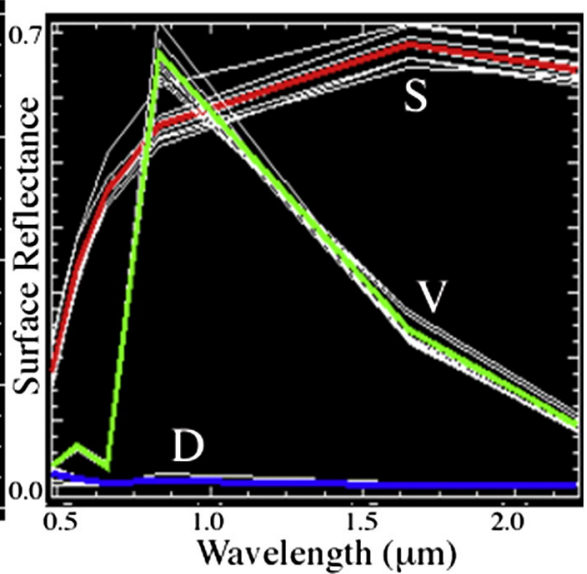

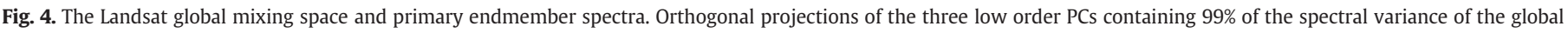

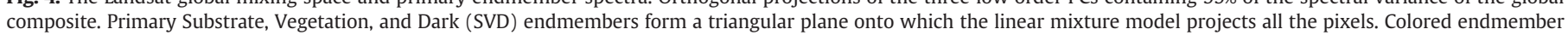
spectra are means of each suite of 9 individual pixel spectra (white). Spectra of secondary endmembers (smaller font) are shown in Fig. 5 . Mean spectra given in Table 1. 
continua with the dark endmember (Figs. 4 and 5). These binary mixtures are generally linear but there is some curvature with water depth in the Lake Eyre subscene. Because ice and snow and shallow marine substrates are not well represented in the global composite, we expect these limbs of the mixing space may not necessarily be representative of the actual spectral diversity of these materials.

The 3D structure of the primary mixing space has a plane of substrates at its base tapering to well defined apexes for the dark and vegetation endmembers. In contrast, the substrate endmember is less well defined, and presents larger variability. The plane of substrates is analogous to the plane of soils (Kauth \& Thomas, 1976) but also includes rock and sediment not typically considered to be soil, as well as non-photosynthetic vegetation such as litter and senescent foliage that has not decayed sufficiently to be incorporated into soil. The primary substrate apex $\mathrm{S}$ is associated with sands near the Richat structure in Mauritania. The secondary substrate endmember s2 (Figs. 4 \& 5) is associated with soils from the Lake Eyre subscene. It has a similar shape to the primary endmember but lower amplitude. The secondary substrate endmember s1, associated with exposed sandstones in the Richat subscene, is considerably lower albedo than the primary endmember but represents the end of a distinct binary mixing line bounding one edge of the plane of substrates. The opposite edge of the plane of substrates does not have a clear apex but rather curves continuously toward the secondary endmember s2. This endmember appears similar to NPV but may also encompass soils and partially decayed NPV. In many cases it is difficult to distinguish NPV from soil spectrally since NPV is often a component of soil. As seen in Fig. 5, the spectra
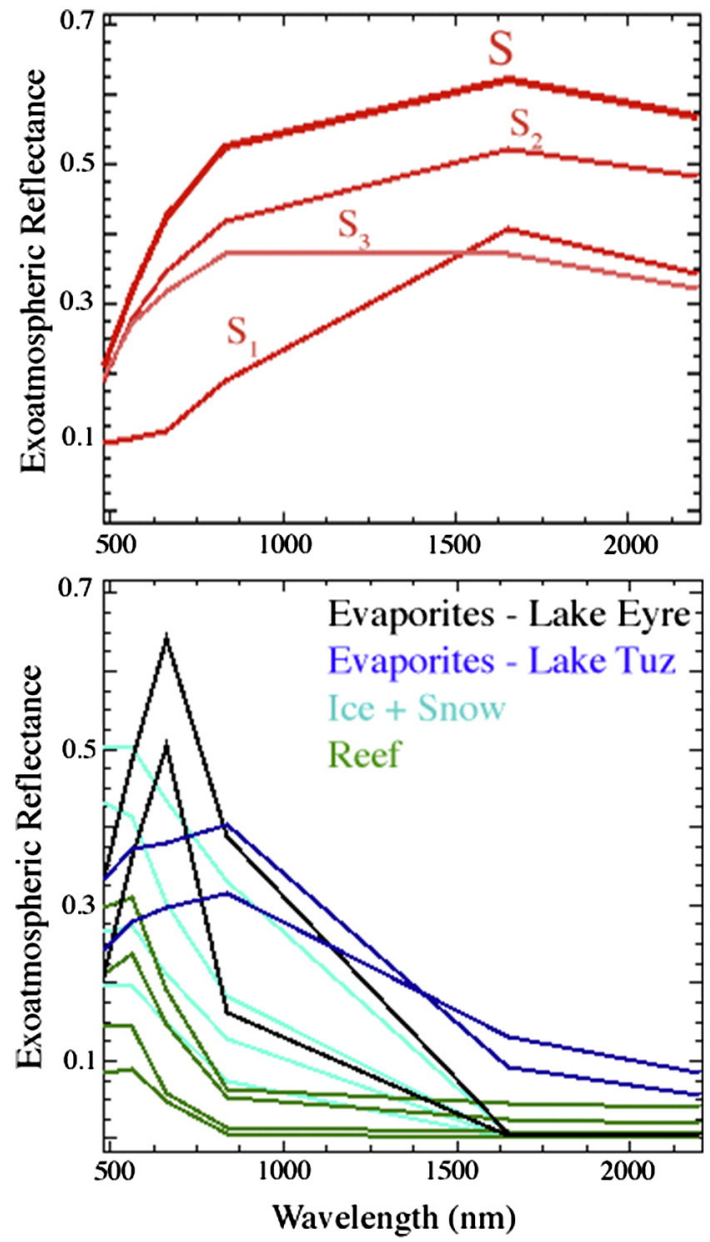

Fig. 5. Binary mixing continua for the plane of substrates and endmembers outside the main SVD hull Substrates (top) form a basal plane on the primary mixing space. Visible-bright spectra (bottom) outside the SVD hull are sampled along linear continua from the Dark endmember.
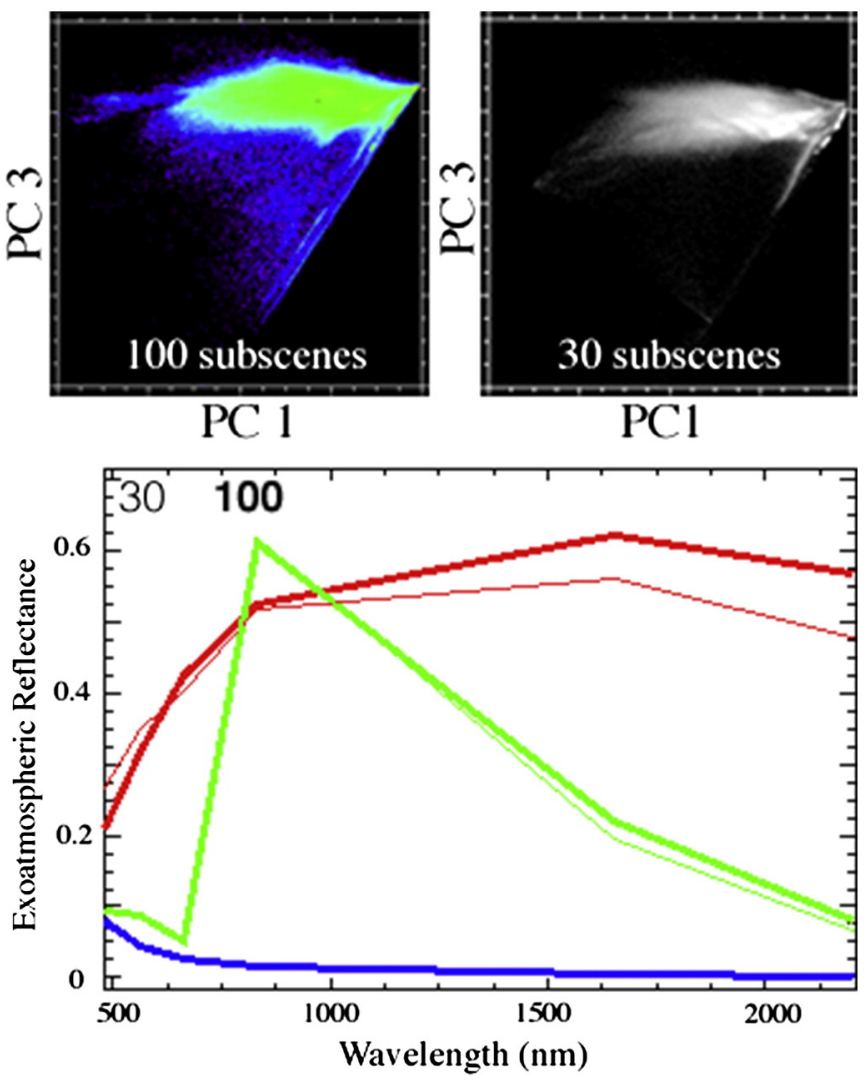

Fig. 6. Exoatmospheric endmembers and the plane of substrates. EMs and mixing space topology of the 30 subscenes used by Small (2004b) are very similar to those from the 100 subscenes in this study. The larger set of 100 subscenes fills out the plane of substrates and converges to a brighter substrate endmember.

along this edge of the plane are more absorptive at SWIR wavelengths. The structure of the plane of substrates and the brighter substrate endmember represents the most obvious difference between the 100 subscene composite used in this study and the 30 subscene composite used by Small (2004b). Fig. 6 shows the effect of extending the plane of substrates with the additional subscenes and convergence to a single endmember somewhat brighter at SWIR wavelengths than that from the 30 subscene composite. The vegetation and dark endmembers are nearly identical for the 30 and 100 subscene composites.

\subsection{Model misfit and fraction sensitivity to endmember variability}

The variability of the primary SVD endmembers is surprisingly low considering the number and diversity of subscenes used in the global composite. Fig. 4 shows the suites of 9 spectra for each endmember (white) and averages of each 9 (color). These potential endmember spectra correspond to individual pixels (or means of $<5$ pixels in a few cases). Because these mean endmembers correspond to small numbers of pixels with nearly identical spectra they are effectively equivalent to single pixel spectra. Overall, endmember variability generally introduces biases less than 0.05 in fraction difference distributions - although for a few combinations of endmembers it increases dispersion as much as 0.4 (Fig. 7). Rather than attempt to justify an ad hoc selection of individual pixel spectra for the global endmembers, we simply use the average of each suite of individual pixel spectra as the global SVD endmembers. All subsequent steps of the analysis use these average endmembers. We average the same individual pixel spectra for both the exoatmospheric and surface reflectance so the resulting endmembers are derived from the same pixel suites and differ only in the correction that has been applied to each.

Unmixing the global composite with the global endmembers gives an indication of the uncertainty expected when applying the standardized 

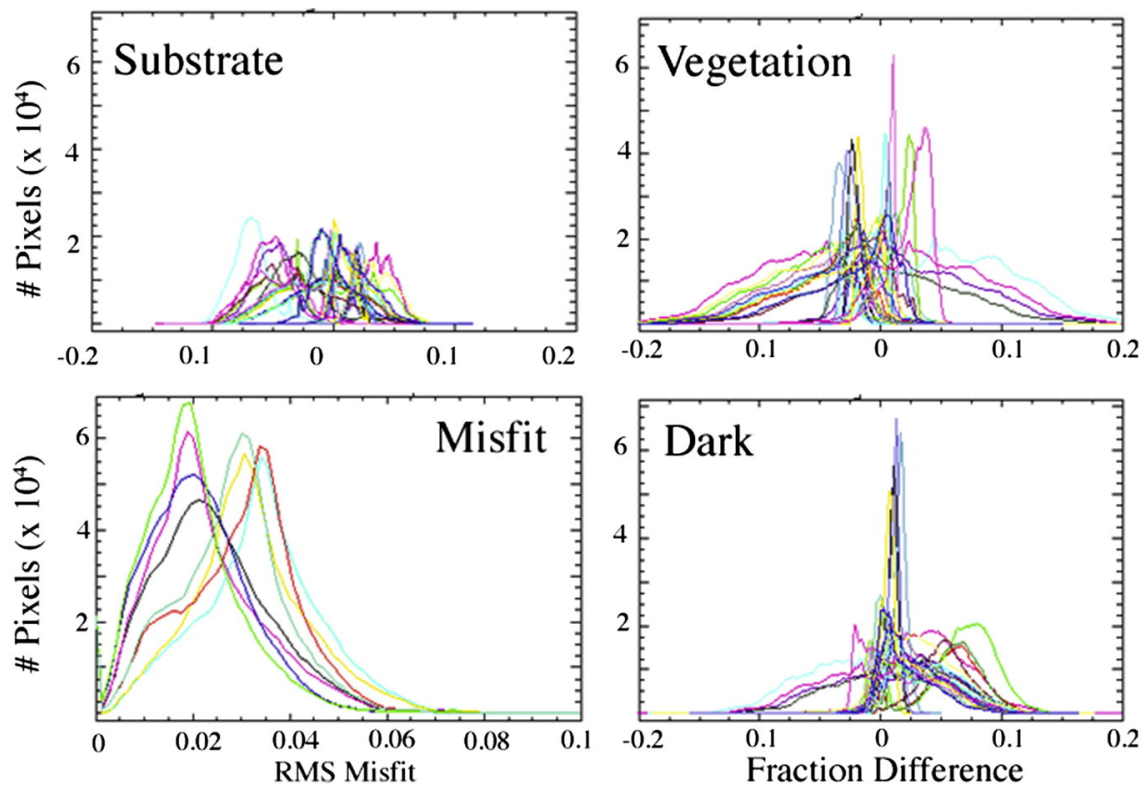

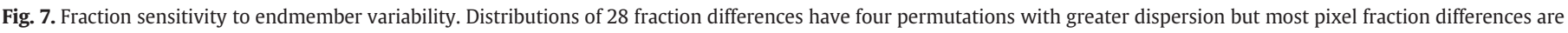
$<0.05$. Misfit distributions for all models are generally $<0.06$.

mixture model with global endmembers to Landsat imagery in general. Fig. 8 shows the RMS misfit distribution and its relationship to the resulting SVD fraction estimates for the global composite. Consistent with the variance distribution given by the eigenvalues, and the use of the average endmembers to define a planar triangular hull bisecting the $3 \mathrm{D}$ pixel cloud, almost all pixels (97\%) have misfits $<0.05$. Prominent exceptions are the pixels on the Visible-bright continua that fall outside the SVD model. As expected, misfit distributions show increasing misfit for intermediate fraction mixtures near the plane of substrates and diminish monotonically toward each endmember. This is consistent with
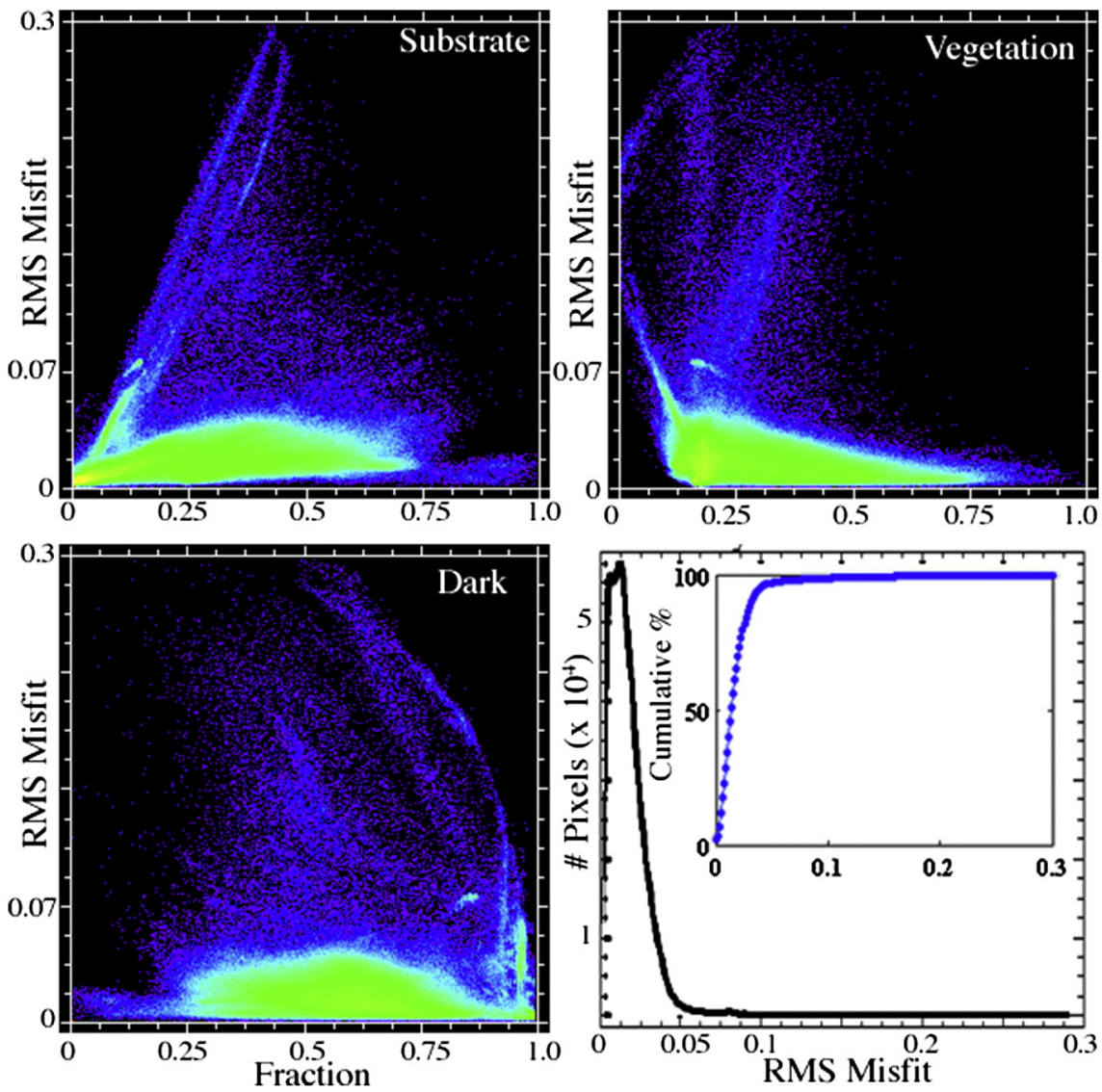

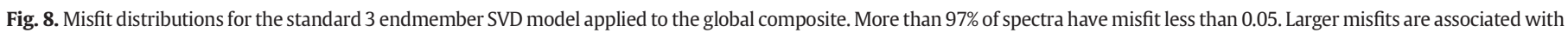
spectra outside the SVD hull (shallow marine substrates, hydrous evaporites, snow and ice). 
the greater 3D dispersion of the mixing space on the plane of substrates. A maximum of $2 \%$ of pixels have negative fractions $<-0.1$ for the Dark fraction with fewer negative fractions for the other two endmembers. The infeasibility of negative fractions is a result of model overfitting and illustrates the difference between actual areal fractions (or spectral contributions in the case of non-linear mixing) and the statistical estimates given by the model inversion.

\subsection{Linearity of scaling}

We investigate both scaling and portability of the global SVD model by comparing fractions derived from similar endmembers at different spatial resolutions. The similarity of the spectral responses of the VNIR bands of Landsat and WV2 suggests that the global endmembers derived for Landsat might be applicable to WV2 imagery (Fig. 9). Indeed, image endmembers for the WV2 scene are similar to the Landsat global endmembers for the Dark and Vegetation endmembers. Not surprisingly, the Substrate endmembers are different (Fig. 9). The WV2 substrate endmember corresponds to impervious surfaces not resolved by the Landsat sensor. Comparing 8-band WV2 exoatmospheric reflectance unmixed with local image endmembers to 6-band Landsat ETM+ surface reflectance unmixed with global SVD endmembers verifies the linearity of scaling of each fraction from $2 \mathrm{~m}$ to $30 \mathrm{~m}$. Using two similar but distinct SVD models at two different spatial and spectral resolutions with two different calibrations introduces several potential sources of variability and therefore provides a relatively stringent test of linearity of scaling. Using the same global SVD endmembers for the same exoatmospheric reflectance calibration on both sensors provides a complementary test whether the VNIR bands of the global SVD endmembers can be used to unmix the WV2 reflectances and yield
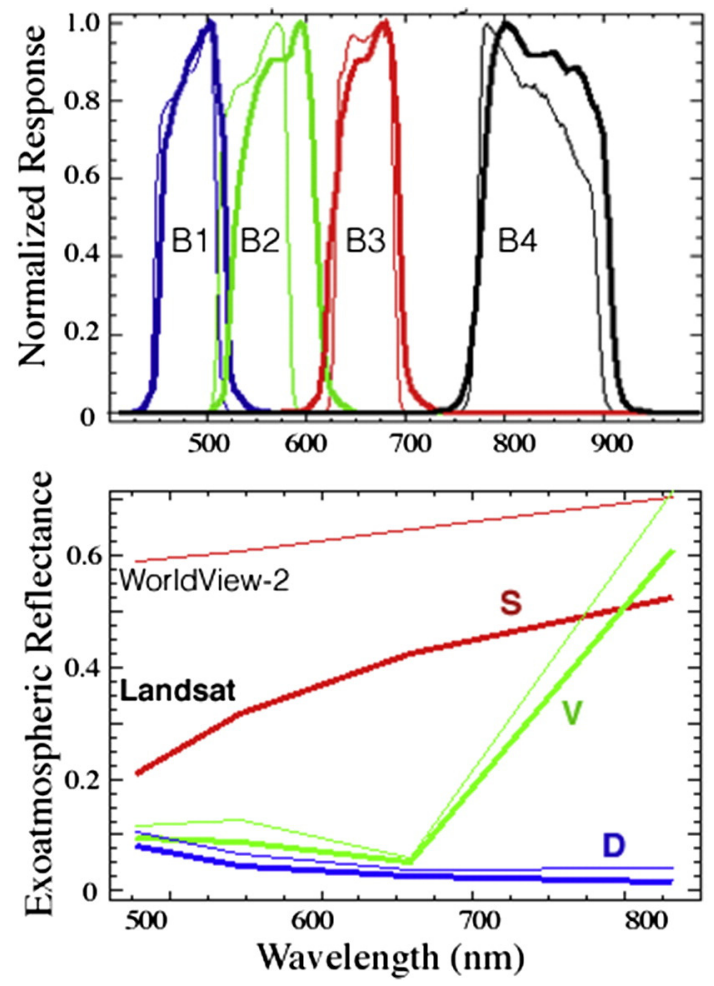

Fig. 9. Spectral responses and exoatmospheric endmembers for WorldView-2 and Landsat TM. Aside from a $\sim 100 \mathrm{~nm}$ shift in the visible green bands, both sensors have similar VNIR responses (top). Local image EMs from NYC WorldView-2 differ from Landsat global EMs primarily in the substrate EM although the red edge of the vegetation endmember spectrum is larger with WorldView-2 (bottom). comparable scaling to the Landsat-derived fractions. Performing both comparisons on two different dates in four different locations with two different Landsat sensors provides a further test of the generality of the SVD model and the linearity of its scaling.

We test linearity of scaling by comparing SVD fractions from global endmembers with high resolution fractions for two pair of coincident Landsat and WV2 acquisitions. We compare coregistered Landsat and WV2 fractions over 4 spectrally diverse test sites in NYC. The Brooklyn site (Fig. 10) encompasses Floyd Bennett Field, some residential areas and part of the Jamaica Bay wetland. The decommissioned airfield (now a park) contains a diversity of marsh grasses and exposed soils. The Bronx site (Fig. 11) encompasses Pelham Bay Park, Eastchester River and wetland, Orchard Beach and part of Long Island Sound. The northwestern part of the site also contains several $20+$ story residential towers. The Manhattan site contains a wide range of combined residential and commercial areas on the Upper East and West Sides and Harlem, and all of Central Park. Most buildings in upper Manhattan are $>5$ stories high and span more than 100 years in age so the area contains a wide variety of impervious surfaces in varying degrees of shadow. The Queens site is more suburban in nature, containing primarily single-family homes and some industrial development. The area contains two large parks, one of which is entirely closed canopy deciduous forest, and a wide range of street tree densities. Although we conducted the analysis for 4 sites, we show only 2 examples here because the results for all 4 sites are very similar. We show the 2 sites with the greatest diversity of land cover in Figs. 10 \& 11 .

Both vegetation and dark fractions show strongly linear scaling from $2 \mathrm{~m}$ to $30 \mathrm{~m}$ in all four test sites for both model comparisons (Figs. 10 \& 11). Using the global SVD endmembers eliminates the slight negative bias of the Landsat vegetation fractions above $\sim 0.2$ at the expense of introducing a slight positive bias for fractions $<\sim 0.2$. Dispersion about the $1: 1$ line does not differ appreciably for the vegetation fractions in any of the 4 sites. For the dark fractions the global SVD endmembers shift the entire range of WV2 fractions from a very slight positive to a very slight negative bias with no appreciable change in dispersion about the 1:1 line. As expected, the substrate fractions do not scale as linearly as the vegetation or dark fractions for either model - although the maximum fraction values are increased somewhat with the global SVD fractions. Given the diversity of substrates in general, and the diversity of impervious surfaces resolved by WV2 in the urban environment, it is not surprising that substrate endmember does not scale linearly between Landsat and WV2. In addition, the ETM + SWIR bands provide additional information not available to the VNIR model used for the WV2 data. The SWIR bands are especially important for distinguishing different substrates because several important mineral and NPV absorptions occur at these wavelengths. For these reasons, we expect that the substrate endmember will be the primary source of model disparity in a variety of environments. In practice, it may be more appropriate to use a location specific substrate endmember with the generic vegetation and dark endmembers.

Having established the linear scaling of the vegetation and dark fractions, we next compare vegetation fractions to other vegetation metrics. Comparing vegetation fractions and metrics using the global composite provides a comprehensive depiction of how different metrics represent a wide variety of vegetation types and abundances in a wide diversity of environments. We compute the Normalized Difference Vegetation Index (NDVI) (Rouse et al., 1973), the Enhanced Vegetation Index (EVI) (Huete et al., 2002), the Soil-Adjusted Vegetation Index (SAVI) (Huete, 1988) and Tasseled Cap greenness (Kauth \& Thomas, 1976) for the $100,000,000$ Landsat spectra in the global composite. Scatterplots of each of these metrics plotted against standardized vegetation fraction estimates are shown in Fig. 12. Strong linear relationships exist between the vegetation fraction estimates and three of the four metrics. Tasseled Cap greenness is strongly linear for fractions $>0.1$ but attains a maximum value of 0.5 for vegetation fractions of 1.0. Tasseled Cap greenness has negative values for 

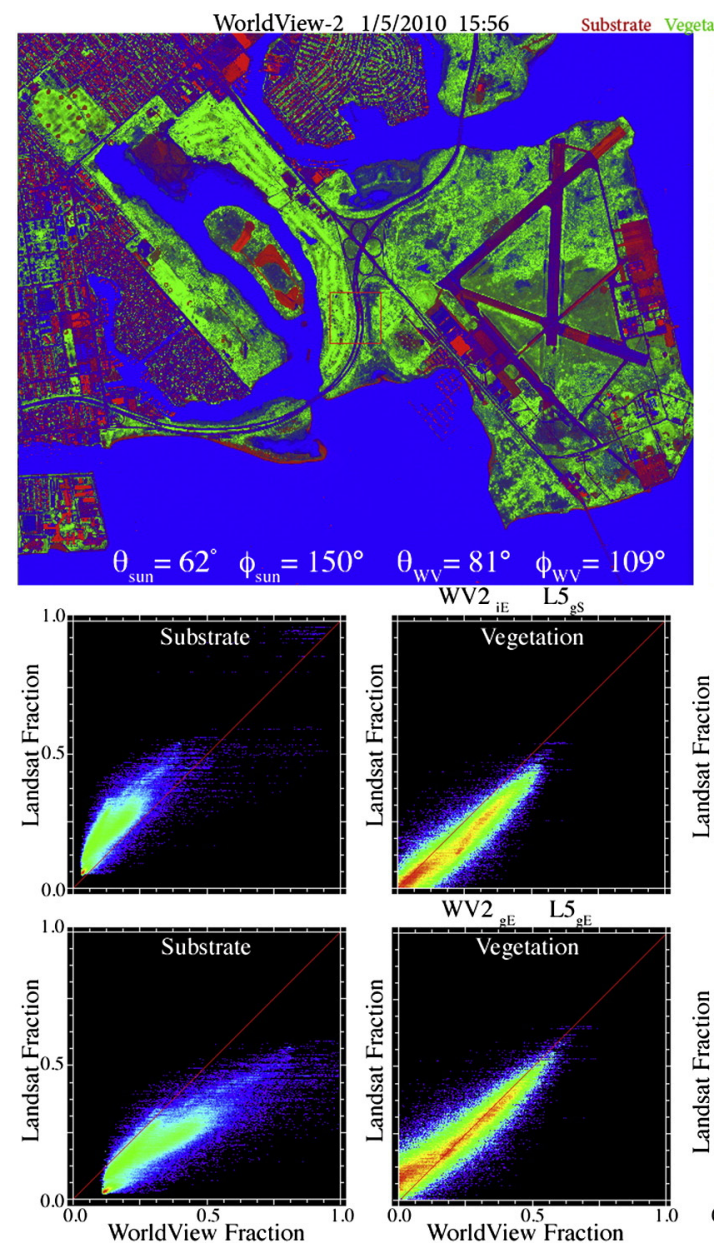

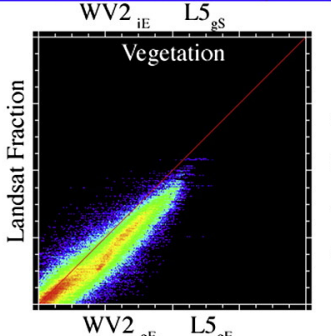

WV2 ${ }_{\mathrm{gE}} \quad$ L5

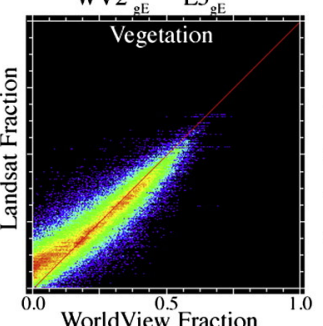

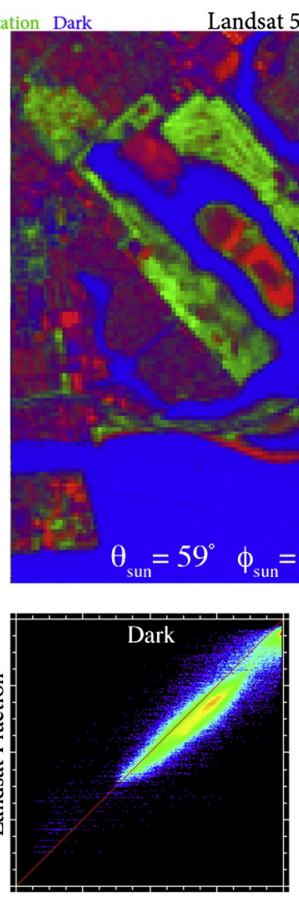
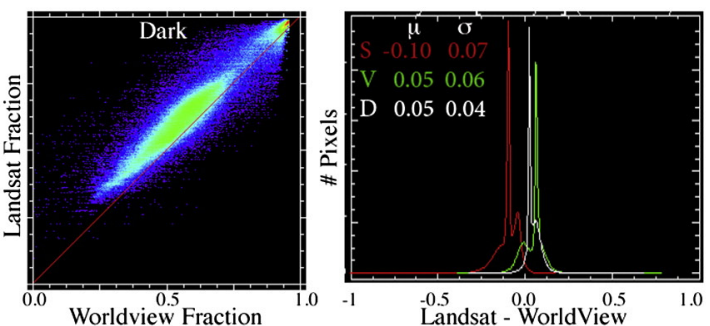

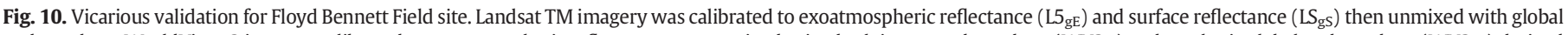

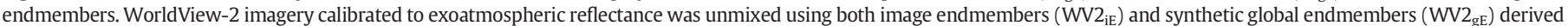
from the Landsat global endmembers. Fraction difference distribution moments quantify the bias $(\mu)$ and the uncertainty $(\sigma)$ of the estimates. Image area is $5.2 \times 4.4 \mathrm{~km}$.

fractions $<0.1$. EVI is strongly linear over the full range of vegetation fractions but has a slight positive bias. SAVI is also linear for vegetation fractions $<0.6$ with some saturation at higher fractions. NDVI shows considerable variability over the full range of vegetation fractions, and begins to saturate at fractions between 0.2 and 0.5 .

\section{Discussion}

To our knowledge, this is the most extensive analysis of the Landsat spectral mixing space completed to date, bringing together into one model a comprehensive representation of terrestrial biomes. The topology of the mixing space of the 100 sites used here has a strong similarity with the space defined with the 30 subscenes analyzed by Small (2004b). This suggests that, except for the plane of substrates, expanding the analysis to additional scenes would likely not further alter the representation of the Landsat mixing space. It is possible, however, that including an even larger set of scenes could additionally alter the plane of substrates but not necessarily influence the primary endmember. The scenes selected here contain very few substrates from urban areas, but previous analyses suggest that most urbanized impervious areas fall within the SVD model identified here (Small, 2005) because of the coarse spatial and spectral resolution of the Landsat sensors and the spectral similarity (and provenance) of many impervious materials to pervious substrates.

This larger variability of the substrate endmember leads, unavoidably, to somewhat greater RMS misfits in the substrate fractions. As discussed in Small (2004b), the RMS may be reduced by including additional substrate endmembers in the standardized spectral mixture model but this would come at the risk of overfitting the model and could produce physically infeasible fractions with negative values. A preferred alternative to reduce the misfit could be to optimize the substrate endmember to the specific scene being analyzed, although there is still the possibility that multiple substrates co-exist within close proximity. In the case where multiple substrate endmembers are present and including both in a single model could lead to instability, it may be appropriate to use multiple endmember spectral mixture analysis as proposed by Roberts et al. (1998).

Using atmospherically corrected surface reflectances versus exoatmospheric reflectances did not alter the shape of the mixing space - likely because the scenes selected for the analysis were all taken under clear sky conditions and were mostly cloud free. However, the fractions derived from surface reflectance data did show less dispersion in the vicarious validation with the WV2 data and in the comparison with the vegetation indices (results not shown). Additionally, the availability of endmembers from atmospherically corrected images allows for more valid comparisons of sequences of SVD fractions over time.

The standardized spectral mixture model is conceptually similar to the Kauth-Thomas Tasseled Cap Transformation (TCT) but differs in two important ways. The TCT is a single rotation (affine transformation) that is applied identically to every pixel while the inversion of the linear mixture model is a linear transformation that varies from pixel to pixel depending on its location within the mixing space. As a result, the linear mixture model effectively orthogonalizes the substrate and vegetation 

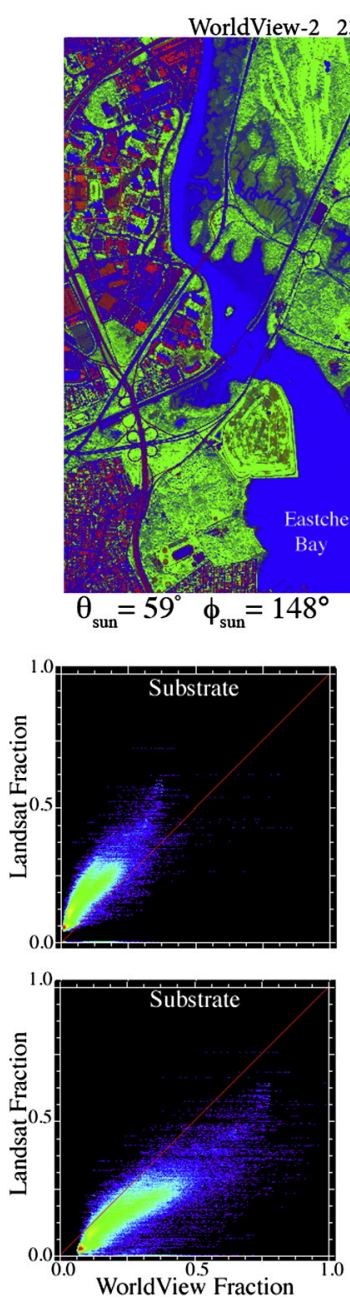

$23 / 4 / 2010 \quad 15: 48$
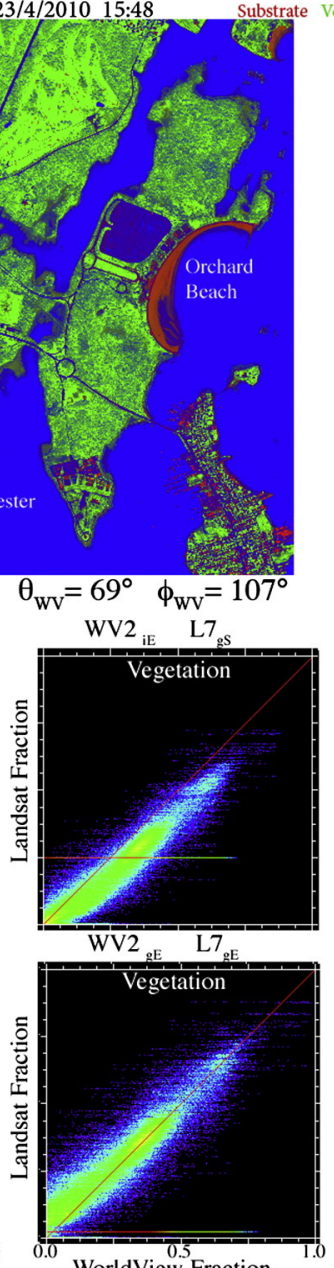

WorldView Fraction
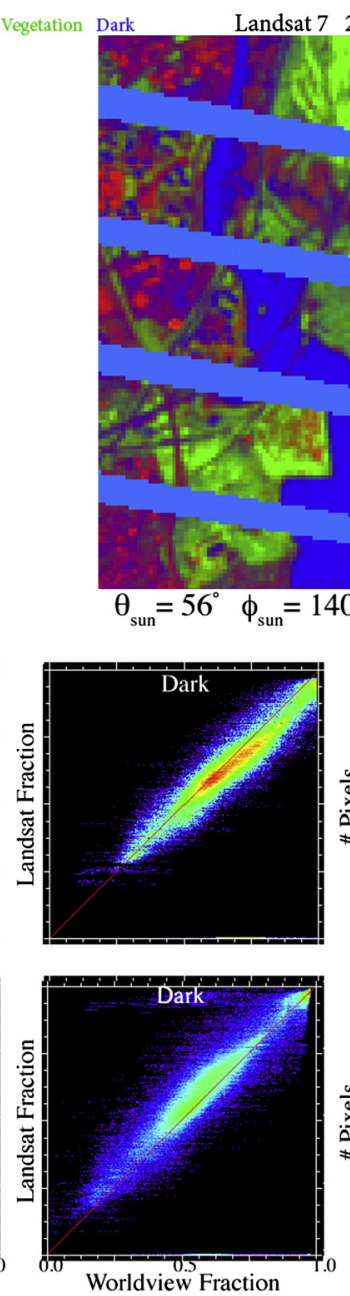

Landsat $7 \quad 23 / 4 / 2010 \quad 15: 25$
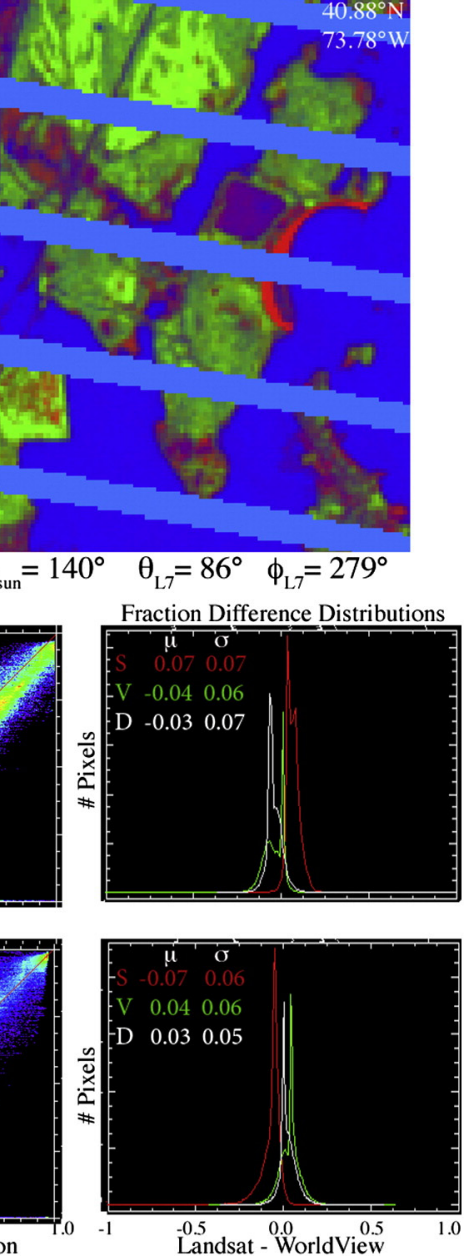

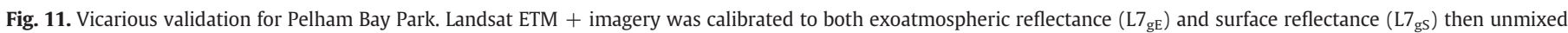

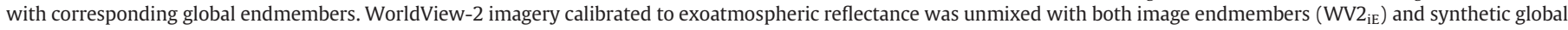

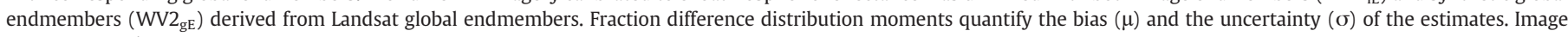
area $4.4 \times 4.4 \mathrm{~km}$.

fractions, overcoming one of the major limitations of the TCT in its inability to separate soil and vegetation contributions to mixed reflectances. This difference is illustrated graphically in Fig. 10 of Small (2004b). Another advantage of the linear mixture model is the ability to customize it with application-specific endmembers which can be represented as combinations of the generic global endmembers. This provides a way to compare application-specific models to the unifying standardized model.

The concept of standardized mixture models and generic global endmembers can be extended to include other broadband sensors with spectral responses similar to Landsat ETM+. In this way, the benefits of the standardized spectral mixture model can be conferred to other sensors without the need to compile calibrated global composites for each sensor. However, the mixing space depicted by the global Landsat composite is only a low dimensional projection of the higher dimensional mixing space that would be resolved by high resolution hyperspectral imagery. The spatial and spectral resolution limit the ability of any broadband sensor to resolve the actual spectral dimensionality of Earth surface materials. Broadband sensors can distinguish general classes of materials with similar spectral responses but cannot resolve narrow band absorptions that distinguish many materials. Therefore, only the low dimensional structure of the mixing space is represented by broadband imagery.

The benefits of standardized mixture models are simplicity, consistency, inclusivity, and applicability. Because the generic endmembers span the mixing space, they provide an alternative "coordinate system" for representing and describing broadband spectra in terms of the fundamental physical components of land cover (and its texture) at decameter scales. Surface texture of land cover at decameter scales results in subpixel shadow manifest as variations in dark fractions. The ability to quantify the shadow component of the reflected radiance field, in addition to the spatial fractions of the illuminated materials, is an important benefit of mixture models not generally available from spectral indices. Temporal change in dark fraction under different solar illuminations can provide potentially useful constraints on surface textures of some land cover types. Temporal change in dark fraction under similar illuminations may suggest changes in surface texture or moisture content. The fact that illumination differences are manifest in the trade-off between the dark fraction and the other endmember fractions suggests an alternative approach to removing terrain-induced variations in illumination and solar incidence angle. Alternatively, dark fractions can be removed by normalizing the complementary fractions (Adams \& Gillespie, 2006). Another important benefit is the linearity of spatial scaling demonstrated in this analysis. Because of the non-linear normalization, ratio indices do not generally scale linearly (Huete et al., 2002) and are known to saturate at higher vegetation density (Carlson \& Ripley, 1997; Choudhury et al., 1994; Dymond et al., 1992; Gillies \& Carlson, 1995). Spectrally unmixed vegetation fractions from Landsat have been found to have excellent correlations with ground fractions of vegetation cover (Elmore et al., 2000). 

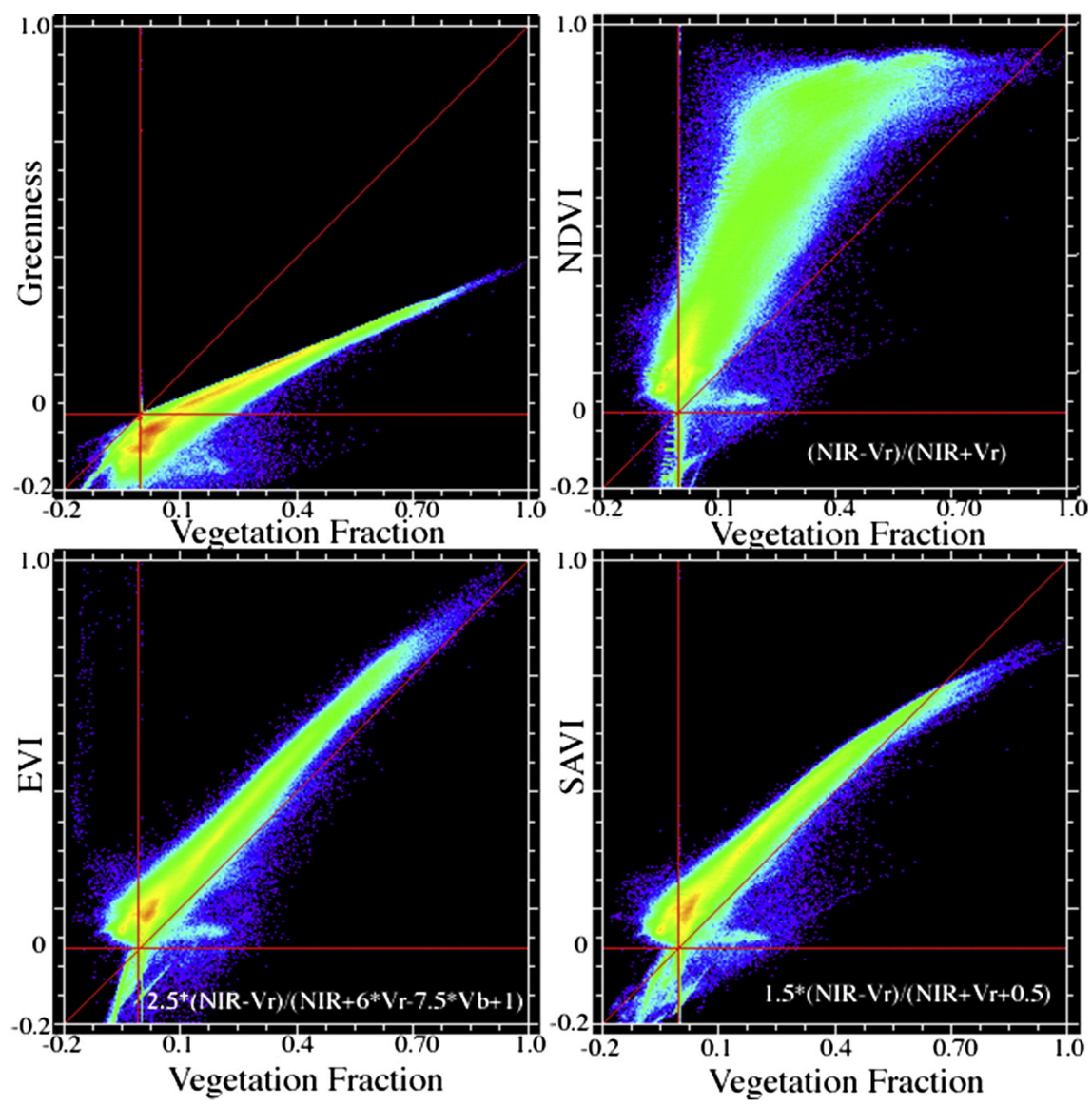

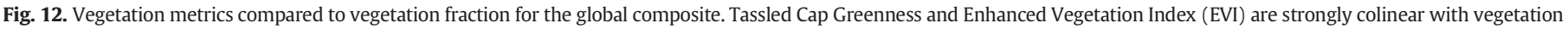

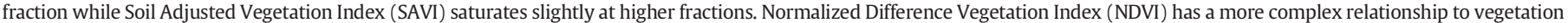
fraction with a wide range of values for all fractions.

Future comparisons of fractions from the standardized model with ground data will help evaluate the utility of this approach for applications that require a consistent regional monitoring of landscape changes at Landsat resolution. The consistency of endmember fractions assocated with specific land cover types may provide a more intuitive alternative to statistical classification in the form of decision tree classifications based on explicitly specified combinations of endmember fractions. The concept of process pathways discussed by Adams and Gillespie (2006) could easily be extended across spatial and temporal scales by incorporating the standardized mixture model. This could provide a physical basis for upscaling and downscaling land surface properties for physical process models. Additionally, the validation of the substrate fractions from the standardized model could provide a basis for monitoring changes in substrate fractions associated with expansions of built-up area caused by urbanization.

It is important to emphasize that we do not advocate the use of the standardized mixture model instead of application specific models but in addition to. For the additional cost of unmixing an image with the global SVD endmembers, application-specific endmember fractions can be compared directly to the global endmember fractions to place them in the context of the full range of spectral diversity imaged by the Landsat sensors.

\section{Acknowledgments}

This study was made possible through funding from the NASA Land-Cover and Land-Use Change (LCLUC) Program (grant NNA07CN16A). We acknowledge the use of the NASA Earth Exchange
(NEX) for the processing of the Landsat data. We thank the anonymous reviewers for the numerous helpful comments and suggestions.

\section{References}

Adams, J. B., \& Gillespie, A. R. (2006). Remote sensing of landscapes with spectral images. Cambridge, UK: Cambridge University Press.

Adams, J. B. Smith, M. O. \& Gillespie, A. R. (1993). Imaging Spectroscopy: Interpretation based on spectral mixture analysis. In C. M. Pieters, \& P. Englert (Eds.), Remote geochemical analysis: elemental and mineralogical composition (pp. 145-166). New York: Cambridge University Press.

Adams, J. B., Smith, M. O., \& Johnson, P. E. (1986). Spectral mixture modeling; a new analysis of rock and soil types at the Viking Lander 1 site. Journal of Geophysical Research, 91, 8098-8122.

Carlson, T. N., \& Ripley, D. A. (1997). On the relation between NDVI, fractional vegetation cover, and leaf area index. Remote Sensing of Environment, 62, 241-252.

Chander, G., Markham, B. L., \& Helder, D. L. (2009). Summary of current radiometric calibration coefficients for Landsat MSS, TM, ETM +, and EO-1 ALI sensors. Remote Sensing of Environment, 113, 893-903.

Choudhury, B. J., Ahmed, N. U., Idso, S. B., Reginato, R. J., \& Daughtry, C. S. T. (1994). Relations between evaporation coefficients and vegetation indices studied by model simulations. Remote Sensing of Environment, 50, 1-17.

Dymond, J. R., Stephens, P. R., Newsome, P. F., \& Wilde, R. H. (1992). Percentage vegetation cover of a degrading rangeland from SPOT. International Journal of Remote Sensing, 13, 1999-2007.

Elmore, A. J., Mustard, J. F., Manning, S. J., \& Lobell, D. B. (2000). Quantifying vegetation change in semiarid environments: precision and accuracy of spectral mixture analysis and the normalized difference vegetation index. Remote Sensing of Environment, 73, 86-102.

Gillespie, A. R., Smith, M. O., Adams, J. B., Willis, S. C., Fischer, A. F., \& Sabol, D. E. (1990). Interpretation of residual images: spectral mixture analysis of AVIRIS images, Owens Valley, California. Proceedings of the 2nd Airborne Visible/Infrared Imaging Spectrometer (AVIRIS) Workshop (pp. 243-270). Pasadena, CA: NASA Jet Propulsion Laboratory.

Gillies, R. R., \& Carlson, T. N. (1995). Thermal remote sensing of surface soil water content with partial vegetation cover for incorporation into climate models. Journal of Applied Meteorology, 34, 745-756. 
Houghton, J. T., Filho, L. G. M., Callander, B. A., Harris, N., Kattenberg, A., \& Maskell, K. (1996). Climate Change 1995: The Science of Climate Change. Cambridge UK: Cambridge University Press.

Huete, A. R. (1988). A Soil-Adjusted Vegetation Index (Savi). Remote Sensing of Environment, 25, 295-309.

Huete, A., Didan, K., Miura, T., Rodriguez, E. P., Gao, X., \& Ferreira, L. G. (2002). Overview of the radiometric and biophysical performance of the MODIS vegetation indices. Remote Sensing of Environment, 83, 195-213.

Ju, J., Roy, D. P., Vermote, E., Masek, J., \& Kovalskyy, V. (2012). Continental-scale validation of MODIS-based and LEDAPS Landsat ETM + atmospheric correction methods. Remote Sensing of Environment, 122, 175-184.

Kauth, R. J., \& Thomas, G. S. (1976). The Tasseled Cap - a graphic description of the spectral-temporal development of agricultural crops as seen by Landsat. Proceedings of the Symposium on Machine Porcessing of Remotely Sensed Data (pp. 4B41-44B51). West Lafayette, Indiana: Purdue University.

Masek, J. G., Vermote, E. F., Saleous, N., Wolfe, R., Hall, F. G., Huemmrich, F., et al. (2006). A Landsat surface reflectance data set for North America, 1990-2000. Geoscience and Remote Sensing Letters, 3, 68-72.

Roberts, D. A., Gardner, M., church, R., Ustin, S., Scheer, G., \& Green, R. O. (1998). Mapping chapparal in the Santa Monica mountains using multiple endmember spectral mixture models. Remote Sensing of Environment, 65, 267-279.
Rouse, J. W., Haas, R. H., Schell, J. A., \& Deering, D. W. (1973). Monitoring vegetation systems in the great plains with ERTS. Third ERTS Symposium (pp. 309-317). NASA.

Settle, J. J., \& Drake, N. A. (1993). Linear mixing and the estimation of ground cover proportions. International Journal of Remote Sensing, 14, 1159-1177.

Small, C. (2004a). Global population distribution and urban land use in geophysical parameter space. Earth Interactions, 8, 1-19.

Small, C. (2004b). The landsat ETM plus spectral mixing space. Remote Sensing of Environment, 93, 1-17.

Small, C. (2005). A global analysis of urban reflectance. International Journal of Remote Sensing, 26, 661-681.

Small, C., \& Lu, J. (2006). Estimation and vicarious validation of urban vegetation abundance by spectral mixture analysis. Remote Sensing of Environment, 100, 441-456.

Smith, M. O., Ustin, S. L., Adams, J. B., \& Gillespie, A. R. (1990). Vegetation in deserts: I. A regional measure of abundance from multispectral images. Remote Sensing of Environment $31,1-26$.

Somers, B., Asner, G. P., Tits, L., \& Coppin, P. (2011). Endmember variability in spectral mixture analysis: A review. Remote Sensing of Environment, 115, 1603-1616.

Vermote, E. F., Tanré, D., Deuzé, J. L., Herman, M., \& Morcrette, J. J. (1997). Second simulation of the satellite signal in the solar spectrum: An overview. IEEE Transactions on Geoscience and Remote Sensing, 35, 675-686. 